Revue d'histoire de l'Amérique française

Ali REVUE D.HISTOIRE DE L'AMÉRIQUE FRANÇAISE

\title{
L'influence spirituelle de Monsieur Henri-Marie Boudon aux origines de notre histoire
}

\section{Anselme Longpré}

Volume 3, numéro 2, septembre 1949

URI : https://id.erudit.org/iderudit/801550ar

DOI : https://doi.org/10.7202/801550ar

Aller au sommaire du numéro

Éditeur(s)

Institut d'histoire de l'Amérique française

ISSN

0035-2357 (imprimé)

1492-1383 (numérique)

Découvrir la revue

Citer cet article

Longpré, A. (1949). L'influence spirituelle de Monsieur Henri-Marie Boudon aux origines de notre histoire. Revue d'histoire de l'Amérique française, 3(2), 200-209. https://doi.org/10.7202/801550ar d'utilisation que vous pouvez consulter en ligne.

https://apropos.erudit.org/fr/usagers/politique-dutilisation/ 


\section{L'INFLUENCE SPIRITUELLE DE MONSIEUR HENRI-MARIE BOUDON AUX ORIGINES DE NOTRE HISTOIRE}

Le nom et les Oeuvres de Henri-Marie Boudon sont actuellement quasi inconnus au Canada. ${ }^{1}$ Dans un article récent, l'abbé $\mathbf{R}$. Heurtevent, aumônier du monastère de la Visitation de Caen, écrivait cependant: "Les livres de Boudon ont eu un succès extraordinaire en France et au Canada" "2. L'histoire prouve en effet clairement que Boudon a exercé la plus grande influence sur la vie intérieure des Fondateurs de notre pays et de leurs disciples immédiats. S'il est permis de parler d'une "spiritualité canadienne", on peut affirmer sans hésiter que Boudon en est le père ${ }^{3}$. Mgr de Laval et Mgr de Saint-Vallier, les Ursulines de Québec et des Trois-Rivières, les Sulpiciens et les Récollets, les Jésuites et nos dévots laiques de cette époque sont tous les disciples fervents de Boudon ${ }^{4}$. Il est facile de présumer que l'influence spirituelle de Boudon se prolongea même après l'âge des Fondateurs, car les livres étant très rares à cette époque, il n'y a pas de doute qu'on conservait longtemps ceux qu'on avait la bonne fortune de posséder.

1. Fn 1941, nous avons édité nous-mêmes, au Centre Catholique de SaintHyacinthe, sous le titre: Le Christianisme, le traité de Boudon: "La Science et la Pratique du Chrétien." En quelques mois, cet opuscule tiré à 10,000 exemplaires était épuisé. Nous ne croyons pas que Boudon ait jamais été édité en dehors de la au Canada.

2. Dictionnaire de Spiritualite, art. Boudon, col. 1892.

3. Par cette expression, nous n'entendons pas restreindre le caractère profondément catholique de la spiritualité de nos Fondateurs ni parler d'une nouvelle Ecole de spiritualité; nous voulons simplement indiquer la manière d'envisager la vie spirituelle aux premiers temps de notre Histoire, les pratiques et les grandes dévotions que nos Fondateurs enracinèrent dans l'áme et les coutumes de nos aieux.

4. Non pas évidemment a l'exclusion de tout autre. Chaque Communauté en particulier a ses maítres et ses modèles. 
Il ne sera pas sans importance pour notre histoire de marquer a grands traits cette bienfaisante influence. Nous avons pour le faire des sources de première valeur, en particulier, les différentes Vies de Boudon ${ }^{5}$, la collection de ses Oeuvres Complètes éditées par Migne en 1856, et les trois cent quatre-vingt-sept lettres authentiques de Boudon où les renseignements abondent ${ }^{6}$.

Actuellement les œuvres de Boudon sont quasi introuvables, aussi bien en Europe qu'au Canada. Le silence qui, pendant plus de deux siècles, se fit sur les grands maîtres de l'Ecole française, enveloppa aussi les œuvres de Boudon. Depuis quelques années cependant cette grande Ecole de spiritualité - la plus grande peut-être de toute l'histoire de l'Eglise - revient à l'honneur. Les Eudistes nous ont donné une excellente édition des œuvres complètes de S. Jean Eudes ? Plusieurs ouvrages du Cardinal de Bérulle et de Monsieur J.-J. Olier ont été réédités et largement répandus. Les Editions du Cerf viennent de nous offrir un beau volume des Lettres du P. Charles de Condren ${ }^{8}$. Quant aux œuvres de St-Louis de Montfort, du P. Surin, du P. Lallemant, du P. Grou, elles sont très répandues de nos jours en tous les milieux; et Dom Jamet, trop tôt disparu, nous a donné une grande partie des œuvres de Marie-de-l'Incarnation, la "Thérèse du NouveauMonde", par ses ardeurs séraphiques, mais la fille spirituelle authentique du Cardinal de Bérulle, l'apôtre du Verbe Incarné, sous le rapport de la doctrine ${ }^{9}$. Espérons que ce renou veau spirituel nous vaudra aussi avant longtemps de bonnes éditions des principaux ouvrages du saint archidiacre d'Evreux ${ }^{10}$.

5. Vie de H.-M. Boudon, par l'abbé Pierre Collet, en tête des Oeuvres Complètes de Boudon (Migne), I: 12-264; L'Homme de Dieu Seul, par Jean Darche. Paris, 1863; H.-M. Boudon ou la folie de la Croix, par Louis d'Appilly (Paris, 1863) et plusieurs autres.

6. Oeuvres Complètes de Boudon (Paris, Migne, 1856).

7. Oeuvres Complètes de S. Jean Eudes (12 vol., Paris, Lethielleux, 1922). 1943).

8. Lettres du P. Charles de Condren, publiées par Auvray et Jouffrey (Paris,

9. Marie de l'Incarnation, Ecrits spirituels et historiques (4 vol., Action Catholique, Québec, 1929, 1930, 1935).

10. Les ouvrages de Boudon, très riches de doctrine spirituelle, laissent souvent a désirer sous le rapport de la forme littéraire. 
Pour nous faire une idée exacte de l'influence de Boudon sur nos origines religieuses, il importe de rappeler d'abord sommairement sa vie et ses activités.

Boudon naquit à la Fève, en Haute-Picardie, le 14 janvier 1624. Son père, Jean Boudon, était lieutenant du gouverneur dans la citadelle, ce qui lui permit de demander à Henriette-Marie de Bourbon, fille de Henri IV, d'être marraine de l'enfant. D'où ses premiers noms d'Henri-Marie. Il fit sa première communion à neuf ans et, peu de temps après, le vœu de virginité perpétuelle. Son premier maitre fut un ecclésiastique nommé Havel. Après avoir suivi ses leçons pendant quelque temps il passa au Collège des Jésuites de Rouen qu'il quitta en 1644 pour étudier la philosophie et la théologie à la faculté de Paris. Il confia alors la direction de sa conscience au P. Bagot, jésuite, le maître de saint Isaac Jogues et du célèbre M. de Lévis, chanoine et archidiacre du Vendemois, et de tant d'autres spirituels de ce temps. Un jour, le jeune étudiant, réduit à la plus extrême pauvreté, demande l'aumône, dans l'église de Notre-Dame de Paris, à un inconnu qui s'y trouvait en prière. Cet inconnu n'était autre que M. de Montmorency, père de Mgr de Laval. Monsieur de Montmorency lui offrit sa maison, ajoutant qu'il y trouverait toutes les facilités pour continuer sa philosophie; il le pria seulement de vouloir bien aider son fils François qui lui aussi commençait les mêmes études. Henri-Marie accepta avec empressement. Les deux jeunes gens se lièrent d'une amitié qui devait durer toute leur vie. Les études philosophiques terminées, Boudon quitta la maison de son bienfaiteur. Poussé par un extraordinaire attrait pour la plus absolue pauvreté, il se retira, avec un petit groupe d'amis, au faubourg Saint-Jacques, pour y mener une vie semblable à celle des Pères du désert, tout en poursuivant ses études théologiques, ou en se livrant, sur les conseils mêmes de l'abbé de Laval, à toutes sortes d'œuvres de miséricorde corporelle et spirituelle. A trente ans, Boudon était encore laïque. Enfin, vaincu par les pressantes exhortations du P. Bagot, il reçut la tonsure, le 4 novembre 1653, des mains du nonce pontifical à Paris, dans la chapelle des Jésuites. Boudon cependant hésitait encore à entrer dans les saints ordres, lorsque l'abbé François de Laval, devenu grand archidiacre d'Evreux, jeta les yeux 
sur lui pour le mettre à sa place, car il brûlait du désir de se consacrer aux missions du Canada. Comme Boudon n'était encore ni prêtre ni docteur, l'abbé de Laval se chargea de lui obtenir ses dispenses de Rome et de lui faire conférer son titre de docteur qu'il n'avait pas pu prendre à Paris, à cause de son indigence. Pour disposer les esprits en faveur de cet inconnu et de cet ascète d'un autre âge, l'abbé de Laval alla l'installer lui-même à Evreux. Le 1er janvier 1655, Mgr Gilles Boutant lui conféra le sacerdoce dans son palais épiscopal d'Evreux.

Il n'entre pas dans notre dessein de raconter en détail la vie de Boudon. Qu'il nous suffise de rappeler ici ce qui est nécessaire pour déterminer l'influence qu'il exerça au Canada. Boudon demeura archidiacre d'Evreux jusqu'à sa mort survenue le 31 aout 1702. Le diocèse d'Evreux était à cette époque dans un état lamentable. Boudon s'appliqua avec un zèle infatigable à le restaurer. Il le parcourut luimême mille fois en tous sens, catéchisant, confessant, multipliant les avis aux pasteurs et aux brebis. Toute sa vie surtout il donna l'exemple des vertus chrétiennes qu'il pratiqua à leur plus haut degré. Il fut l'émule de saint Jean de la Croix et de sainte Thérèse pour l'oraison, de saint Bernard pour l'amour de Marie, de saint François pour la pratique de la pauvreté à laquelle il s'était engagé par vœu. Pendant une partie notable de sa vie, il fut victime de toutes les persécutions, tant à l'intérieur du diocèse d'Evreux où il opérait de si grandes et si salutaires réformes, qu'en dehors du diocèse et jusqu'à Rome ${ }^{11}$. Les Jansénistes surtout lui vouèrent une haine implacable et lui firent une guerre à mort, à cause de son extraordinaire dévotion à la Très Sainte Vierge, de son zèle inlassable à défendre son Immaculée Conception et à répandre la dévotion au Saint Esclavage de Marie, selon l'expression chère à cette époque. Longtemps, en effet, avant saint Louis de Montfort, Boudon avait composé un vaste traité: Le Saint Esclavage de l'Admirable Mère de Dieu. Chose qui surprendra plusieurs de nos contemporains: à cette époque, les Jansénistes considéraient comme leurs pires adversaires Monsieur Olier, Monsieur Boudon,

11. Deux de ses ouvrages même furent censurés à Rome: La Dévotion à l'Esclavage de Marie, et Dieu seul. Un décret de la Congrégation déclara cependant que le premier avait été censuré uniquement à cause des abus que plusieurs faisaient de cette dévotion. Le second qui avait reçu l'approbation de Bossuet fut condamné 26 ans après la publication, à l'époque de la crise du Quiétisme, à cause de certaines expressions qui auraient pu favoriser cette erreur et sur lesquelles quelques-uns auraient pu chercher a s'appuyer. 
saint Louis de Montfort, et tant d'autres qui prêchaient à tue-tête comme on ne l'a probablement jamais fait dans l'Eglise - le détachement de tout le créé, la lutte contre l'esprit du monde, l'amour de Dieu seul, et le culte de la Très Sainte Vierge.

Malgré tant de travaux apostoliques, Boudon fut l'un des plus célèbres écrivains spirituels de son temps, tant par le nombre de ses écrits que par leur valeur. Sans doute les écrits de Boudon n'ont pas l'originalité puissante de ceux du Cardinal de Bérulle ou de Monsieur Olier. Boudon n'est pas un fondateur d'Ecole. Mais il fut le plus authentique et le plus puissant vulgarisateur de la doctrine des grands maitres de l'École française. Il connaît leurs écrits quasi par cœur; il les cite constamment, et ne parlent de ses maitres qu'en les nommant "des séraphins terrestres, des prodiges de science et de vertu, des hommes tout divins", etc., etc. Il avait en si haute estime le P. Surin qu'il écrivit sa vie. Il était en relation constante avec les disciples du $P$. Lallemant. Ses conseillers furent le célèbre M. de Lévis, à Chartres; le P. Bourdoise, grand apôtre du clergé, à Paris; Jean de Bernières, disciple du fameux Père Jean Chrysostome, l'âme de cet incomparable mouvement de spiritualité, et qui plus que tous les autres fut le véritable maître de Boudon. Sous le titre: l'Homme Intérieur, Boudon écrivit même le récit de sa vie. Boudon connaissait aussi à fond les écrits de saint François de Sales, de saint Jean de la Croix - dont il écrivit la vie - de sainte Thérèse. Il cite sans cesse ces trois grands maîtres. On peut dire que Boudon nous livre en résumé ce qu'ils ont écrit de plus utile sur la vie spirituelle. Par là, il est facile d'entrevoir de quelle qualité fut l'influence de Boudon. Sans doute Boudon a son originalité propre très marquée. Il fut le précurseur de la dévotion aux Sacrés Cours de Jésus et de Marie; nul peut-être n'a insisté comme lui sur l'importance de la dévotion aux saints Anges dans la vie spirituelle; c'est lui aussi qui traça la voie à saint Louis de Montfort. Mais, avant tout, Boudon est le témoin de la tradition spirituelle catholique.

Comme tous les livres de Boudon ont été répandus à profusion au Canada, il est utile d'en donner les titres et d'indiquer autant que pos- 
sible la date de leur publication ${ }^{12}$. Lui-même, afin que l'on ne s'y méprenne pas, a dressé la liste de ses ouvrages dans une lettre adressée a la mère Elisabeth de Sainte-Marie. "Voici, dit-il, la liste des livres que la Divine Providence nous a fait donner au public: Dieu seul (1662). L'amour de Jésus au Très Saint-Sacrement (1662). - Le règne de Dieu en l'oraison mentale (1671). - Les Saintes Voies de la Croix (1671). De l'amour de Notre-Seigneur Jésus-Christ (1672). - La vie cachée avec Jesus en Dieu (1676). - La conduite de la Divine Providence (1678). - La science sacrée du catéchisme (1684). - Les grands secours de la Providence par Notre-Dame-du-Remède (1684). - L'Homme intérieur ou la vie du P. Jean Chrysostome (1684). - La science et la pratique du chrétien (1681 et 1685). - Dieu présent partout (1690.) Dieu inconnu (1690). - De la profanation des églises. - De la sainteté de l'état ecclésiastique. - La dévotion à la Sainte Trinité. - Le malheur du monde. - La gloire de la Sainte Trinité dans les âmes du Purgatoire. - La dévotion a l'Immaculée Mère de Dieu (1699). — La dévotion aux neuf chœurs des bons Anges. - La vie du P. Surin. - La Vive Flamme d'amour ou la vie de S. Jean de la Croix. - Le triomphe de la Croix ou la vie de Mère Marie Elisabeth. - Avis catholiques touchant la dévotion à la Mère de Dieu. ${ }^{13}$ La vie de S. Taurin, apôtre d'Évreux. - La dévotion a l'Ange Gardien. - La dévotion de l'esclavage de Marie. - La dévotion au règne de Dieu. - Le chrétien inconnu.Les cérémonies de la Sainte Messe.

Les titres à eux seuls nous indiquent déjà que Boudon traite à peu près de tous les sujets intéressant la vie spirituelle.

\section{- III -}

Mgr de Laval avait M. Boudon en très haute estime. Ecrivant à M. Thomas, conseiller au Châtelet - et qui plus que tout autre fut

12. Boudon ne date ordinairement ses livres et ses lettres que de la fête du saint dont l'Église fait mémoire, sans indiquer l'année. Les éditeurs, en examinant les circonstances, ont pu les dater approximativement. Heureusement, l'un des principaux correspondants de Boudon, en ce qui concerne les affaires du Canada, M. Thomas, out soin d'ajouter de sa main, les dates sur les lettres qu'il recevait. Il a rendu ainsi un précieux service à l'histoire.

13. Après avoir dresse cette liste que Boudon croyait finale a cause de ses nombreuses infirmités qui lui faisaient entrevoir sa fin comme prochaine, il eut encore lo temps et ls force d'écrire quelaues autres ouvrages. 
le dépositaire des sentiments les plus intimes de Boudon et le plus infatigable propagandiste de ses livres - il le félicitait "de ce qu'il était à la portée de visiter à Evreux le tombeau de ce cher défunt", ajoutant l'éloge le plus complet que l'on puisse faire de lui, en disant que "sa vie a été une parfaite imitation de celle de Jésus-Christ. Plaise à Dieu de me faire la grâce de l'imiter aussi parfaitement que je l'honore" 14.

Boudon apprit avec une joie profonde de Mgr de Laval lui-même, dans une lettre personnelle, que "la dévotion à la Très Sainte Vierge, à Saint Joseph et aux Saints Anges que ses livres avaient inspirée à tous au Canada avait sauvé Québec de l'amiral Phips"; et le saint prélat, après l'en avoir vivement remercié, terminait sa lettre en marquant une fois de plus son estime pour Boudon: "Je suis toujours, le peu de jours qui me restent a vivre, tout a Vous, en l'amour de Jésus, Marie, Joseph, de tous les Anges et de tous les Saints"1 5.

Boudon avoue lui-même avoir reçu une lettre des plus élogieuses à l'endroit de ses livres, de la part de Mgr de Laval. Il écrit, en effet, à M. Thomas: "Mgr l'évêque de Québec m'a écrit la lettre la plus obligeante du monde au sujet des livres que vous avez eu la charité de lui présenter de ma part; il ne se peut rien trouver de plus humble ni de plus honnête que ce prélat". (Lettre CCCLIII).

Quand Mgr de Laval eut résigné son poste, Mgr de Saint-Vallier continua la même attitude à l'égard des écrits de Boudon. Le 11 février 1702, Boudon écrit à son propagandiste, M. Thomas: "Il ne faut pas manquer d'écrire au Canada que c'est par l'ordre de Mgr l'Evêque que vous envoyez et donnez ces livres aux communautés, car on n'y doit lire que les livres qu'il trouve à propos". (Lettre CCCLIX)

Si Mgr de Laval estimait Boudon, il faut dire que cette estime était réciproque. Boudon écrit à M. Bosguerard: "Le saint homme, le P. de Brébœuf est l'apôtre du Canada; il a souffert des tourments dont la seule idée fait trembler. Il a donc fallu aussi que le premier évêque du Canada ait à porter de pesantes croix. Son zèle, l'empêchant d'entrer dans tous les desseins des Gouverneurs, lui a attiré des grandes

14. M. Boudon, ayant fait le vœu de pauvreté, s'en remettait à ses deux amis, M. Thomas et M. Courtin pour l'impression et la diffusion de ses livres. Ceux-ci s'y appliquèrent avec un zèle incroyable et répandirent les Oeuvres de Boudon dans les cinq parties du monde, mais surtout en France et au Canada.

15. Vie de Boudon, par Pierre Collet, Oeuvres Complètes, I: 147. 
persécutions... cependant Mgr l'Evêque actuel de Québec est un homme de Dieu qui avait refusé des évêchés en France... il pensait glorifier Dieu en ce qu'il a fait au Canada". (Lettre CCXLV)

Boudon suit de près les événements du Canada. Il conseille aux Ursulines de s'établir aux Trois-Rivières: "Les Ursulines m'ont écrit du Canada pour un nouvel établissement qu'elles font, à qui l'on s'oppose, parce que l'homme ne voit pas assez de secours humain..." (Lettre CCXCIII). Apprenant l'incendie du Séminaire de Québec, il écrit à son ami, M. Thomas: "Je ne savais pas les conduites de la Divine Providence sur le Séminaire de Québec; c'est un effet singulier de la même Divine Providence qu'on ait pu retirer les papiers de M. Gardelet..." (Lettre CCCLXI). Dans une autre lettre, il fait l'éloge de Marguerite Bourgeoys et de ces jeunes filles qui, sans être religieuses, se consacrent à l'instruction des sauvages. (Lettre CCCLV)

La diffusion des écrits de Boudon et la faveur dont ils jouissent au Canada nous est attestée maintes fois, soit dans les lettres qua Boudon reçoit, soit dans celles qu'il écrit. Le 19 janvier 1\%01, Boudon écrit à son propagandiste, M. Thomas: "J'ai reçu hier une lettre du Canada; l'un des grands vicaires m'écrit que notre bon Sauveur répand sa bénédiction sur les livres que sa miséricordieuse providence m'a fait donner au public; il me dit qu'il les a distribués aux deux séminaires de Québec et de Montréal, au Collège de Québec, à la grande congrégation, au couvent des Récollets de Québec, aux deux maisons de l'hôpital général de Québec et de Montréal, aux deux monastères des Ursulines de Québec et des Trois-Rivières. Il souhaiterait que vous continuassiez cette œuvre de Dieu pour quantité d'autres maisons ou assemblées dans le Canada qui n'en ont pas eu. Il ajoute que ces livres sont merveilleusement goûtés par les bonnes âmes. Si, Monsieur, vous croyez devoir faire cette œuvre, il faudrait en faire un paquet et le faire porter à $M$. Tremblay, procureur du Canada, au Séminaire des Missions Etrangères, et je pense que notre bon Sauveur serait glorifié si vous y mettiez un bon nombre de promesses de soutenir l'Immaculée Conception de Notre-Dame..." (Lettre CCCXXII)

Dans d'autres lettres, Boudon énumère les livres qu'on lui demande et ceux qu'il fait envoyer par M. Thomas. (Lettres CCCXXIV; CCCXXV, etc.,) On constate alors que toutes les Oeuvres de Boudon franchirent les mers pour venir alimenter la piété au Canada, non 
seulement dans les cloîtres, mais aussi "auprès des personnes qui vivent dans le siècle". (Lettre CCCXLIX) Sans cesse harcel6 de demandes, il peut y satisfaire, malgré son extrême indigence, grâce à la générosité de ses deux propagandistes, M. Thomas et M. Courtin.

A plusieurs reprises, nous trouvons dans ses lettres à $M$. Thomas des formules comme celle-ci: "Je vous envoie un paquet de lettres pour le Canada... Voici un paquet de lettres pour le Canada..." (Lettre CCCLIV; CCCLIX, etc., etc.)

Ces témoignages auxquels nous aurions pu ajouter plusieurs autres sont suffisants pour établir le bien fondé de la parole de Collet: "Il se faisait un débit incroyable des livres de Boudon au Canada"; et l'estime dont ils jouissaient.

Il est facile aussi de discerner l'influence des écrits de Boudon, a l'origine des grandes dévotions populaires de nos aïeux.

Dès l'origine de notre Histoire, l'Immaculée Conception de Marie fut l'objet d'un culte solennel et d'une dévotion particulière. Mgr de Laval voulut même consacrer à l'Immaculée Conception l'église cathédrale qu'il fit bâtir à Québec, en 1666.

Dans un Bref du 14 juin 1892, Léon XIII, faisant l'historique du culte de la Sainte-Famille, rappelle que ce culte "s'est développé en Amérique, dans la région du Canada, où il devint très florissant, grâce principalement à la sollicitude et à l'activité du vénérable serviteur de Dieu, François de Montmorency Laval et de la vénérable Servante de Dieu, Marguerite Bourgeoys". Tous les deux avaient puisé ce culte surtout dans les écrits de Boudon.

Nous ne croyons pas qu'jl y ait eu dans toute l'histoire de l'Eglise un plus ardent apôtre de la dévotion aux Saints Anges et en particulier a Saint Michel que l'archidiacre d'Evreux. Pas un écrit, pas une lettre, sans qu'il en soit question. "Je voudrais que ces paroles du grand St Léon, pape: "Confirmate amicitias cum sanctis angelis; faites des amitiés avec les Saints Anges", parussent en toutes les avenues des villes et des villages, en toutes nos églises, dans toutes les chambres et les cabinets de travail; que partout il y eat des personnes dont le grand soin fat de les redire; que tous les prédicateurs ne fissent jamais aucun sermon, sans les publier fortement et qu'elles fussent toujours insinuées dans les entretiens particuliers" ${ }^{16}$. Ces recommandations

16. Dewotion aux neuf Chours des Anges. Prologue. Oeuvres, II: 831. 
portèrent leur fruit au Canada. La dévotion à Saint Michel pénétra profondément l'âme de notre peuple. Les propriétaires prenaient ordinairement possession de leurs nouveaux domaines à la Saint-Michel. C'était la date que l'on choisissait souvent pour le payement des dettes. Les encans d'une certaine importance se tenaient a la SaintMichel. Dans bien des cas, les fiançailles, les mariages ou d'autres óvénements importants de la vie sociale s'accomplissaient en cette fête. Dès 1680, un petit sanctuaire lui fut dédié à Percé, par les soins du frère Didace Pelletier, récollet. En 1693, une paroisse, Saint-Michel de Bellechasse, est érigée sous son patronage, et dans la suite un grand nombre d'autres le choisirent comme patron spécial.

Ces quelques notes suffisent a établir que le nom de Henri-Marie Boudon doit figurer à côté de celui de $\mathrm{Mgr}$ de Laval, de $\mathrm{M}$. Olier et de Marie de l'Incarnation, parmi les grands bienfaiteurs de notre pays. Ce nom qui était sur toutes les lèvres et dans tous les coours, au commencement de notre histoire, ne doit pas être oublié de la géneration actuelle.

Anselme LongrRe, Ph., L;Th.,L.

Curé de Ste-Marie-de-Brigham, P.Q. 